\title{
The role of the mesentery in reducing recurrence after surgery in Crohn's disease
}

\author{
Dakshitha Wickramasinghe $^{1,2}$ (D) Janindra Warusavitarne ${ }^{1}$
}

Received: 31 December 2018 / Accepted: 4 January 2019 / Published online: 2 February 2019

(C) Italian Society of Surgery (SIC) 2019

Crohn's disease $(\mathrm{CD})$ is a relapsing and remitting disorder with an annual incidence of 3-20 per 100,000. Approximately, half of the patients will have the disease affecting the terminal ileum and a third will have isolated small bowel disease. The probability of requiring surgery for $\mathrm{CD}$ is $78 \%$ and $90 \%$ after 20 and 30 years of disease, respectively [1]. Post-operative recurrence is an important issue in ileocolic disease, with an estimated re-operation rate of $11-32 \%$ at 5 years increasing to $46-55 \%$ at 20 years [2]. Post-operative treatment goals usually centre on minimising disease recurrence.

Crohn's disease has been thought to originate in the gut lumen, and on this premise, the medical treatment tends to focus on treating luminal inflammation [1]. On this basis, surgical treatment has involved a conservative approach to the mesentery as opposed to cancer surgery where removal of the mesentery and associated lymph nodes is essential. Crohn identified changes in the mesentery in his seminal paper, but these have largely been thought to be secondary to gut inflammation. Recently, the small bowel mesentery has received more attention from the observation that the extent of the mesentery excised with the affected intestine affects the rate of recurrence after surgery [1].

Although CD was believed to originate in the intestine and the mesenteric changes were thought to be secondary, researchers have identified mesenteric abnormalities on MRI in patients who had not developed any mucosal changes on endoscopy [3]. Additionally, mesenteric hypervascularity, which may present as a "comb sign" on computed tomography, is strongly predictive of subsequently developing $\mathrm{CD}$. There has been good topographic correlation between mucosal and mesenteric disease extent, including similar

Dakshitha Wickramasinghe

dakshithaw@srg.cmb.ac.lk

1 Department of Surgery, St. Mark's Hospital, London, UK

2 Department of Surgery, Faculty of Medicine, University of Colombo, Colombo, Sri Lanka transition zones [1]. In addition, there has been good correlation noted between the $\mathrm{CD}$ activity index (CDAI), mucosal disease activity and mesenteric disease activity [1].

Histologically, there have been similarities noted between the changes in the mesentery and the submucosa [3]. They both show hyperplasia of adipocytes and thickening of connective tissue. The transmural nature of the disease may be a combined effort of simultaneous disease activity intra- and extra-luminally [3]. This is further supported by the observation that, although inflammation and oedema are circumferential (and likely caused by the changes in the submucosa), ulceration is predominantly at the mesenteric pole (and likely driven by the mesentery) [3].

There is additional indirect evidence to implicate the mesentery as the driver of the disease process in CD. Stricturoplasty is a common procedure for $\mathrm{CD}$ strictures and does not include removal of any bowel or mesentery. Complete mucosal healing is often seen at sites of stricturoplasty, and only $2.8-3.7 \%$ will have a recurrence at or near the stricturoplasty site [2]. This supports the hypothesis that nonmucosal factors may be the driving force behind recurrence in CD after surgery.

Excision of the mesentery is automatically associated with an increased lymphatic tissue removal. This may reduce the immunological stimulation and result in improved postoperative outcomes [1]. It may also reduce the recruitment of fibroblasts which subsequently migrate to the intestinal surface [1]. A mesenchymal input may be behind both mesenteric and mucosal changes.

The findings of the study by Coffey et al., however, should be interpreted with some caution, as they have used a historical cohort for comparison. This group included 30 patients operated over 7 years. Smoking was also disproportionately higher in the group that had a conventional resection. A randomized controlled trial is currently underway, and preliminary results are similar to those observed by Coffey et al. [1].

One of the main reasons surgeons attempt to divide the mesentery close to the intestine is that the Crohn's mesentery 
is thick and bleeds easily when divided. As a result, the surgeon needs to be extra cautious in dividing the mesentery and achieving haemostasis. Given the mounting evidence, an extensive mesenteric resection with meticulous haemostasis could become the standard of care in small bowel resection for CD.

Like the small bowel mesentery, complete removal of the mesorectum by dissecting in the mesorectal plane (vs close rectal dissection) reduces the pro-inflammatory immune status in the surrounding tissue in CD. No such difference in the immune status was noted in patients with ulcerative colitis (UC). This had also translated to less perineal complications in patients with CD [4]. The patients who underwent a total mesorectal excision for $\mathrm{CD}$ had similar rates of perineal complications and healing, as the patients having a proctectomy for UC. The histology of the mesorectal tissue identified a skewing towards the myeloid population between $\mathrm{CD}^{+}$and $\mathrm{CD} 14^{+}$cells. There was also a reduction in the expression of regulatory markers on the macrophages. The mesorectal tissue in CD had high expression of TNF $\alpha$ mRNA, whereas it was undetectable in patients with UC. All these factors support removing the fatty tissue of the mesentery along with the bowel segment in patients with $\mathrm{CD}$ requiring a proctectomy.

Despite this, the paper by Kono et al. [5], which describes a novel anastomotic technique for use in patients with CD, contradicts this theory. In this technique, the excision of the strictured segment is performed close to the mesenteric edge, preserving the mesentery of the excised segment. The divided edges of bowel are sutured together to create a supporting column which isolates the mesentery from the anastomosis. A functional end-to-end anastomosis is then performed. Despite leaving the entire mesentery behind, the authors report a significantly lower endoscopic and surgical recurrence rate using this technique and it is possible that this could be the result of isolating the mesentery from the anastomosis. The rate of recurrence even for patients not receiving biologics was lower than patients who received biologics after resection of the mesentery and undergoing a conventional anastomosis.

Assuming the mesentery is the driver of inflammation in $\mathrm{CD}$, one possible explanation for these contradicting reports could be the existence of different phenotypes of $\mathrm{CD}$ which remain to be identified and classified: a mesentery-dependent and mesentery-independent disease types. Alternatively, the response of the mesenteric fat cells and the immune mediators in the mesentery could have a different response to the same stimulus. A major confounder is that patients with perforating disease will always have a resection. It is only in structuring the disease that we see both treatment options being used.

There is evidence to support that the disease behaviour in $\mathrm{CD}$ remains largely unchanged in recurrence. If this holds true to the mesenteric behaviour model, once the phenotype is identified, an informed decision could be made on future interventions [6].

\section{Compliance with ethical standards}

Conflict of interest None declared.

Research involving human participants and/or animals This article does not contain any studies with human participants or animals performed by any of the authors.

Informed consent No informed consent is required.

\section{References}

1. Coffey JC et al (2018) Inclusion of the mesentery in ileocolic resection for Crohn's disease is associated with reduced surgical recurrence. J Crohns Colitis 12(10):1139-1150

2. Yamamoto $T$ (2005) Factors affecting recurrence after surgery for Crohn's disease. World J Gastroenterol 11(26):3971-3979

3. Coffey JC et al (2016) The mesentery in Crohn's disease: friend or foe? Curr Opin Gastroenterol 32(4):267-273

4. de Groof EJ et al (2018) Persistent mesorectal inflammatory activity is associated with complications after proctectomy in Crohn's Disease. J Crohn's Colitis. https://doi.org/10.1093/ecco-jcc/jjy13 1

5. Kono $\mathrm{T}$ et al (2011) A new antimesenteric functional end-to-end handsewn anastomosis: surgical prevention of anastomotic recurrence in Crohn's disease. Dis Colon Rectum 54(5):586-592

6. Simillis $\mathrm{C}$ et al (2008) A meta-analysis comparing incidence of recurrence and indication for reoperation after surgery for perforating versus nonperforating Crohn's disease. Am J Gastroenterol 103(1):196-205

Publisher's Note Springer Nature remains neutral with regard to jurisdictional claims in published maps and institutional affiliations. 